\title{
An Adaptive Method Based on High-Level Petri Nets for E-Learning
}

\author{
Fatemeh Omrani $^{1 *}$, Ali Harounabadi ${ }^{2}$, Vahid Rafe ${ }^{3}$ \\ ${ }^{1}$ Department of Computer Engineering, Arak Branch, Islamic Azad University, Arak, Iran; ${ }^{2}$ Department of Computer Engineering, \\ Central Tehran Branch, Islamic Azad University, Tehran, Iran; ${ }^{3}$ Department of Computer Engineering, Faculty of Engineering, Arak \\ University, Arak, Iran. \\ Email: *fa_omrani@yahoo.com
}

Received August 31 ${ }^{\text {st }}, 2011$; revised September $23^{\text {rd }}, 2011$; accepted October $15^{\text {th }}, 2011$.

\begin{abstract}
Adaptive learning is a new approach for e-learning systems. In comparison to traditional e-learning systems, which present same things for all learners, these systems automatically adapt with learner characteristics. In this paper, we are going to propose a new method for Adaptive learning, and consider adaptation from three viewpoints: 1) learner learning style; 2) learner's knowledge level; 3) learner's score. Due to similarity between learning objects graph and petri net, and In order to provide adaptive learning, we use an approach based on a high level petri net (HLPN). Also we propose a method to evaluate performance in this system. We compare our system with a non adaptive system, through our performance evaluating method. The results show response time for our system is less than non adaptive system and learners finish course in a relatively shorter period of time. Since our proposed system considers individual features of learner, we can be sure that learner would not be confused in learning materials.
\end{abstract}

Keywords: Adaptive Learning, Learning Style, High Level Petri Nets, Response Time

\section{Introduction}

In e-learning systems, learners are faced with considerable amount of information in different format. If all information is presented to learners, it would result in two problems: First, learners would be confused with wide informational world; and the second, the needs of the user do not satisfy. So these problems result in educational failure and lack of motivation in obtaining knowledge. So designing adaptive e-learning systems is an important issue in e-learning field. Adaptive e-learning environments are environments, which provide learning materials based on individual learner features. Adaptive learning environments ensure to provide effective and efficient learning for learners. Dahbi et al. classified adaptation into two categories [1]: 1) adaptive presentation; 2 ) adaptive navigation. The former indicates adaption in content level. In other words, there are some features like details of presentation, media type, etc. that, in different situations, affect the presented content and result in having different presented content. The latter means adaptation the web links between learning objects for each learner, is adapted by system automatically. In our proposed method, both techniques are considered. Because the presented learning material which is available for learner, not only due to content, is adaptive with user's characteristics; but also due to background knowledge level and his/her score, the path which he/she travels, can be different. Most methods that consider knowledge level for adapting course [2,3]; ignore learner's improvement in recent course. In proposed method, learner improvement is assessed by checking leaner's score. Checking this factor increases learner's performance and arriving to his/her learning target in shorter time. For separating learner from individual attribute we use color token in color petri net. Also, for evaluating performance, we use token with time stamp in timed petri net.

Many researchers have conducted researches in adaptive learning field and have used different method for adaptive learning. For example, Semet et al. used an artificial intelligence approach, called" ant colony optimization" [4]. In this system ants are presumed as learners, and adaptive learning path is made by considering pheromone which is released by other learners. De Marcos et al. proposed a PSO algorithm for presenting adaptive link to user [5]. In this method, sequencing problem of the learning object for learner, is resembled to permutCSP problem and to solve this problem; used an agent which 
works based on PSO. Zhu et al. used a learning activity graph and allocated Boolean expression to every edge of graph [6]. This expression includes required precondition and post condition for traveling through this edge. If this expression is evaluated correctly, corresponding edge is traversed by learner. Chen et al. used "Item response theory" for providing individual learning path for each learner [7]. Manouselis et al. used multi-criteria decision making for automatic presentation of learning path in personalized environment [8].

Regarding similarity between learning object graph and petri net, some methods in adaptive learning field use various types of petri net. A dynamic fuzzy petri net (DFPN) was used for increasing flexibility of tutoring agent [9]. Tutoring agent is auxiliary software for helping individual user. Based on individual user behavior, the tutoring agent presents a different structure of learning content. Chang et al. used a Behavioral Browsing model (B2model) based on High level petri net (HLPN) which was made for modeling and generating behavioral pattern of students [10]. Object oriented course modeling based on High level petri net (HLPN) was proposed by Su et al. [11]. And one authoring tool was made based on proposed model. Liu et al. proposed an approach based on petri net for controlling learning path among learning activities [12]. Different learners learn in different ways. For example, someone learns by observation, someone learns by listening, someone learns by doing practices, someone learns by concentrating on principle, someone learns by concentrating on application, someone persists on "memorization" and someone prefers understanding. To provide adaptive learning some other researches have focused on learning style [13-15], but these methods are not formal and don't evaluate performance by executable model. In comparison we use learning style factor for adaptation, and propose an executable model by using formal method.

We use three factors for adaptation: 1) knowledge level; 2) learning style [16]; and 3) score. And we should add that none of the previous methods based on Petri net, such as [2,3], use learning style for adaptation; whereas this is a key factor in learner satisfaction. So we should say that this is an advantage for our method to consider it. In addition, in our system, we proposed a method to evaluate performance and we evaluated our system by it. Results show, by decreasing response time; performance increases. In evaluation section of this paper, we demonstrate in this system, learner with low knowledge levelby considering recent score by system can improve her/ his performance. Also we assert if system doesn't use this method, decrease learner performance at high knowledge level and this would increase displeasure.

The following sections are: Section 2 which explains definitions and primary concepts. In Section 3 we express the proposed method. In Section 4 we describe a case study. And then, we evaluate proposed method in Section 5. Finally, we finish paper with conclusion and future work in Section 6.

\subsection{Definition and Primary Concepts}

Petri net-its primitive idea is presented by Carl Adam Petri in $60 \mathrm{~s}$ - is a graphical and mathematical tool that these mentioned characteristics make it easy to use and easy to present [17]. Figure 1 shows a simple example of petri net.

A petri net is a 5 -tuple $[10,17]$ :

$$
P N=\left(P ; T ; A ; W ; M_{0}\right),
$$

where

1) $P=\left\{p_{1}, p_{2}, \cdots, p_{m}\right\}$ is a finite set of places. A place represents a circle, such as, $p_{1}, p_{2}$ and $p_{3}$ in Figure 1.

2) $T=\left\{t_{1}, t_{2}, \cdots, t_{n}\right\}$ is a finite set of transitions. A transition represents a bar, such as $t_{1}$ in Figure 1. The intersection of $P$ and T is an empty set, while the union of $P$ and $T$ is not an empty set, i.e., $P \cap T=\phi$ and $T \cap P=\phi$.

3) $A \subseteq(P \times T) \cup(T \times P)$ is a set of arcs connecting places and transitions, such as the arrowhead from $p_{1}$ to $t_{1}$ depicted in Figure 1.

4) $W: A \rightarrow\{1,2,3, \cdots\}$ is a weight function, whose weight value is positive integers. Arcs, i.e., arrowheads, are labeled with weights. For example, in Figure 1, the arrowhead from $t_{1}$ to $p_{3}$, which is labeled with 2 , is denoted as $W\left(p_{2}, t_{1}\right)=2$. When the weight is unity and/or 1 , the label of arc is usually omitted, e.g., $W\left(p_{1}, t_{1}\right)=1$ is omitted in Figure 1.

5) $M_{0}: P \rightarrow\{0,1,2,3, \cdots\}$ is the initial marking. If there are $k$ tokens inside place $p_{i}$, it is said that $p_{i}$ is marked with $\mathrm{k}$ tokens. For example, in Figure 1(a), $p_{1}$ is marked with one token, which is denoted as $M\left(p_{1}\right)=1 . p_{2}$ is marked with two tokens, which is denoted as $M\left(p_{2}\right)=2$. If Figure 1(a) is the initial status, the initial marking is denoted as $M_{0}\left(p_{1}, p_{2}, p_{3}\right)=1,2,0$.

A transition $t$ is said to be fired if all its input places pi are marked with at least $W\left(p_{i}, t\right)$ tokens, where $W\left(p_{i}, t\right)$ is called the firing condition of transition t. For example, in Figure 1, the firing conditions of $t_{1}$ are $W\left(p_{1}, t_{1}\right)=1$ and $W\left(p_{2}, t_{1}\right)=2$. A firing transition $t$ removes $W\left(p_{i}, t\right)$ tokens

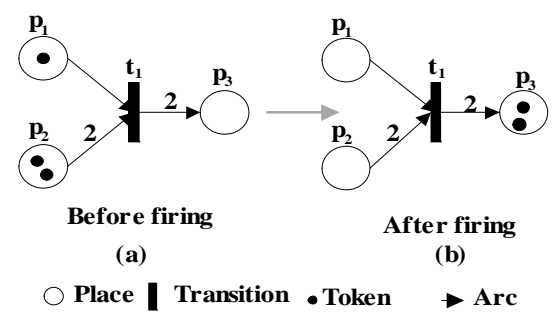

Figure 1. A petri net example. 
from each input place $\mathrm{p}_{\mathrm{i}}$, and adds $W\left(t, p_{j}\right)$ tokens to each output place $p_{j}$. For instance, since $M\left(p_{1}\right)=1$ and $M\left(p_{2}\right)=$ 2 have satisfied the firing conditions of $t_{1}$ in Figure 1(a), $t_{1}$ is fired. After $t_{1}$ is fired as Figure 1(b) depicts, $t_{1}$ has removed $W\left(p_{1}, t_{1}\right)=1$ token from input place $p_{1}$ of $t_{1}$ and $W\left(p_{2}, t_{1}\right)=2$ tokens from input place $p_{2}$ of $t_{1}$, respectively, and then added $W\left(t_{1}, p_{3}\right)=2$ tokens to output place $p_{3}$ of $t_{1}$.

In the following years later, extensions of petri net such as Colored petri net and timed petri net are made, which is called High level petri net(HLPN). In following, we describe these petri nets.

\subsubsection{Colored Petri Net}

In colored petri net a value so called color is allocated to each token. Transitions use the color of input tokens for determining the color of output tokens. Relation between input tokens and output tokens is presented by color consumption function or arc function that is depicted by $E$. for example in Figure 2, E functions are $E_{f}\left(p_{1}, t_{1}\right)=a$, $E_{f}\left(t_{1}, p_{2}\right)=b$. If a transition is fired, colored tokens removed from input places and new color tokens are produced and placed in output places. In Figure 2(a), place $p_{1}$ is marked with two colored tokens $a, b$ from a color set $\{a, b, c\}$, denoted $m\left(p_{1}\right)=\{a, b\} . t_{1}$ is enabled as its input arc only requires one colored token a which is available in place $p_{1}$. If fired, token a is removed from $p_{1}$, two new colored tokens $\mathrm{b}$ and $\mathrm{c}$ are generated by $t_{1}$ 's output arcs and deposited in $p_{2}$ and $p_{3}$, respectively, as shown in Figure 2(b).

\subsubsection{Timed Petri Net}

Timed Petri nets have many types. In this paper, we use a kind of petri net in which each token is corresponded with a realvalued clock that is represented to token age $[18,19]$. Firing condition of transitions is like regular petri net. In addition, each arc which connects a transition to a place is labeled with real number which presents amount of age growth after transition firing. When a transition is fired, age of each token which has been added to output place of transition should be increased. Figure 3 shows a timed petri net. Each token labeled with a real number representing its age. Figure 3(a) shows the age of token in input place $p_{1}$, that is 2 . As shown in Figure 3(b), after firing $t_{1}$, age of token which is added to output place $p_{2}$, changes to 5 .

\subsection{Four Learning Style}

In a web based tutoring system, there is a user interface which its properties are similar to teacher's, who provides learning materials for learner. These materials may have different styles such as audio, video and text or combination of these styles. Drago et al. classify all these styles in four categories and called them $(V A R K)$ styles [16]. So, the place which is shown to learner should be appropriate with his/her learning style. By referring to user's profile, we can extract the specific style that learner prefers it more than the other style. These styles are illustrated in Table 1. In our proposed model, we use colored token for determining learner's learning style. To summarize implementation, we declare a 2 member set: " $V$ " as visual and " $R W$ ' as Read/Write style.

\section{Proposed Adaptive Method}

In our method, each learning object in learning object graph [20], which can be a chapter, a section or an example, is mapped to a place in petri net. Colored tokens are learners, and they travel through learning object via transitions. Color of token checked by transitions and if condition is true, is allowed to move to the next node. So by this method, adaptive path is constructed.

At the beginning of the path, we use initial node and check the learner's learning style in this node by guard function of transition which is connected to this node. And according to individual value of learning style, we guide learner to individual path. Then, in each path, according to background knowledge level and obtained score, we decide the next node.

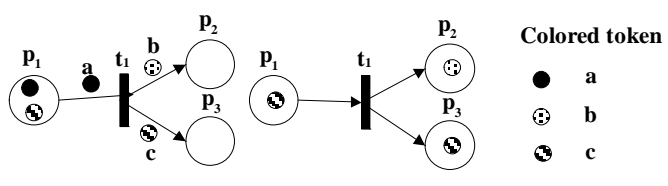

$\begin{array}{ll}\text { (a)Before } t_{1} \text { fires } & \text { (b)After } t_{1} \text { fires }\end{array}$

Figure 2. Colored petrin net.

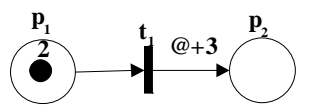

(a) Before $t_{1}$ fires

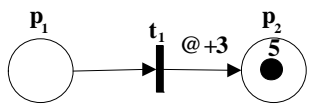

(b) After $t_{1}$ fires
Figure 3. Timed petri net.

Table 1. The vark style [15].

\begin{tabular}{ccc}
\hline $\begin{array}{c}\text { VARK } \\
\text { Learning way }\end{array}$ & $\begin{array}{c}\text { Information is best } \\
\text { absorbed with the use of }\end{array}$ & $\begin{array}{c}\text { Learning } \\
\text { way }\end{array}$ \\
\hline Visual & $\begin{array}{c}\text { Charts, graphs, flow charts, } \\
\text { symbols, arrows, hierarchies }\end{array}$ & $\begin{array}{c}\text { Learn by } \\
\text { seeing }\end{array}$ \\
Audio & $\begin{array}{c}\text { Lectures, verbal tutorials, tapes, } \\
\text { group discussion, speaking, } \\
\text { talking things through }\end{array}$ & $\begin{array}{c}\text { Learn by } \\
\text { hearing }\end{array}$ \\
Read/write & $\begin{array}{c}\text { Any text-based input and } \\
\text { output }\end{array}$ & $\begin{array}{c}\text { Learn by } \\
\text { processing text }\end{array}$ \\
& Experience or practice activity, \\
Kinesthetic & Learning \\
& by doing
\end{tabular}


In our method, we use token with time stamp for calculating response time. Also, we need to calculate the browsing time in which a learner remains in a learning unit, the time that a learner undertakes an assessment and the time that a student remains in queue. Leaner arrives to system according to a Poisson process. So the time LT, at which the next learner will arrive is derived according to Poisson distribution [21] which is

$$
L T=\frac{\mathrm{e}^{\lambda t}(\lambda t)^{n}}{n !}
$$

where $t$ refers to the time that a student has stayed in queue before using system; $\lambda$ refers to the rate of entrance, and since $L T$ is used to determine the time when a learner arrives, we have $n=1$.

The browsing time that a learner stays in a learning unit is derived according to normal distribution [21], which is

$$
B T=\frac{\mathrm{e}^{\frac{-1}{2}\left[\frac{A V G_{B}-\alpha}{\delta}\right]^{2}}}{\sqrt{2 \pi} \delta},
$$

where $A V G_{B}$ refers to the average length of time that a learner stays in a learning unit; $\alpha$ is the variation of time spent in a learning unit among learners; and $\delta$ represents the standard deviation of time spent in a learning unit among learners.

The time that a learner is involved in answering and completing test question is derived according to normal distribution [21], which is,

$$
A T=\frac{\mathrm{e}^{\frac{-1}{2}\left[\frac{A V G_{A}-\beta}{\rho}\right]^{2}}}{\sqrt{2 \pi} \rho},
$$

where $A V G_{A}$ refers to the average test time; $\beta$ is the variation of time spent in a test node among learners; and $\rho$ represents the standard deviation of time spent in a test node among learners.

The score that a learner has earned from a learning unit which has test; derived according to normal distribution [21], which is,

$$
\text { Score }=\frac{\mathrm{e}^{\frac{-1}{2}\left[\frac{A V G_{S}-\mu}{\gamma}\right]^{2}}}{\sqrt{2 \pi} \lambda},
$$

where $A V G_{S}$ refers to the average score of a learning unit which has test; and $\gamma$ represents the standard deviation of a learning unit which has test; and $\mu$ is the variation of score in a learning unit which has test among learners.

We add an integer number to time stamp of new learner who enters to the system which represents her/his arrive time. This integer number is generated with Poisson distributed function as we express in Equation (2).
After that, for each learner who browses a learning unit we add a BT time which is computed via Equation (3). If a learning unit has test, we add a BT time which is computed via Equation (3) and an AT time which is computed via Equation (4) $(A T+B T)$. At the end of the course for obtaining response time, we subtract arrive time $(L T)$ from resent model time for each learner.

In learning units (nodes) which have test we assigned a Score which is computed via Equation (4) to each learner who browses that learning unit. This score is one of the factors which are used for deciding to guide learner to the next unit.

We compute response time in two viewpoints of white and black box. In viewpoint of black box, we calculate only response time for each learner and show it in output text file. Whereas, in viewpoint of white box, in addition to response time, we show traveled path, time spent for each middle learning unit, earned score in each test, learning style and knowledge level for each learner.

We need several monitors for computing response time [22]. A monitor is a mechanism in CPN tool $[23,24]$ which is used to observe, check and control simulation without changing petri net.

In addition to colors, which show user's features; we need several extra colors for computing response time. These colors are: 1) the arrive time of learner, i.e., the time at which the learner arrived in the system; 2) the total amount of time that learner remains in system which is initialized to zero; 3) the learner path that represents unit which learner met them and initialized to null value; 4) the detail time that represents time spent in each unit of learning path.

\subsection{Response Time in Black Box}

We use a "Data collector monitor" for computing response time from the viewpoint of black box and associated it with final transition in course model. In other words, the response time for a learner is measured when the token that is representative of the learner, is added to the final place of the model. After the learner finishes conclusion section of course; the observation function for the monitor, measures the total response time for learner. When final transition is fired, the total time for the learner is bound to the one color of token, and arrive time of token is one of token colors too. So the response time for learner is subtraction arrive time from total time.

\subsection{Response Time in White Box}

We use a "write in file monitor" for computing response time in viewpoint of white box and associate it with final transition in course model. The observation functions for the monitor print of all colors of each token and their response time in output text file. 


\section{Case study}

There are many tools for implementation of petri nets. We use CPN tool for simulation of our proposed model.

For designing course, we consider a book as depicted in Figure 4. This book includes 2 chapters (content 1, content 2), 2 sections (content 1.1, content 2.1), 6 examples, introduction and conclusion sections. The Learning objects graph [25], which is corresponded with this book is shown in Figure 5. We can map this graph to petri net by method which is expressed in [3]. The generated petri net is depicted in Figure 10 and Figure 12. Three basic color sets should be declared for our method in CPN tool: 1) learning style; 2) knowledge level; 3) score. In addition, we need 4 extra color sets: 1) color set, LT, which is represented in arrive time; 2) color set, PATH, which is represented in traveled path by learner; 3 ) color set, Detail Time, which is represented time spent in each middle unit in the course; 4) Process Time in which the total amount of time is represented that the learner stays in system. Since the learner includes all of these features (colors), we declare a compound color LEARNER which is the product of all of these colors. Also, we declare a "list color set", named "Learners" for maintaining learners in a FIFO queue when they are entered in the system. So, declaration of color set in CPN tool, is the one like Figure 6. As it is said in section 3, Leaner arrives to system according to a Poisson process. So, we use the arri-

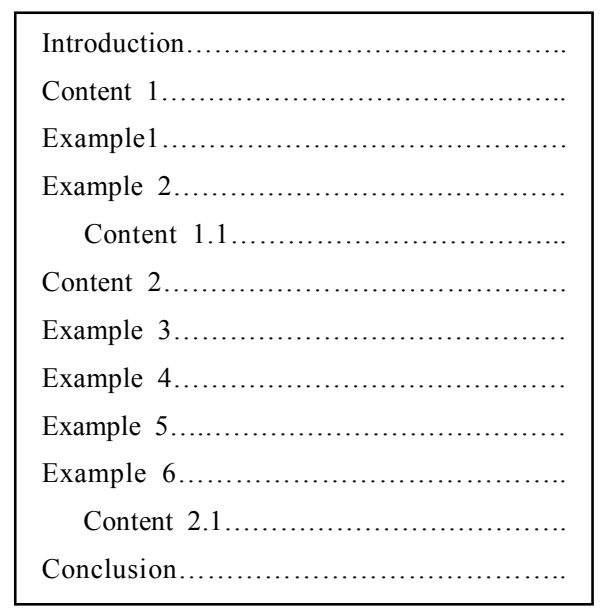

Figure 4. A sample of book structure. vals subpage to model the arrivals of new learners as depicted in Figure 7. The time stamp of the token on next place in the Arrivals page determines the time at which the next learner will arrive. The Poisson function is used here to generate Poisson distributed in inter-arrival times with an average inter-arrival time of 120 .

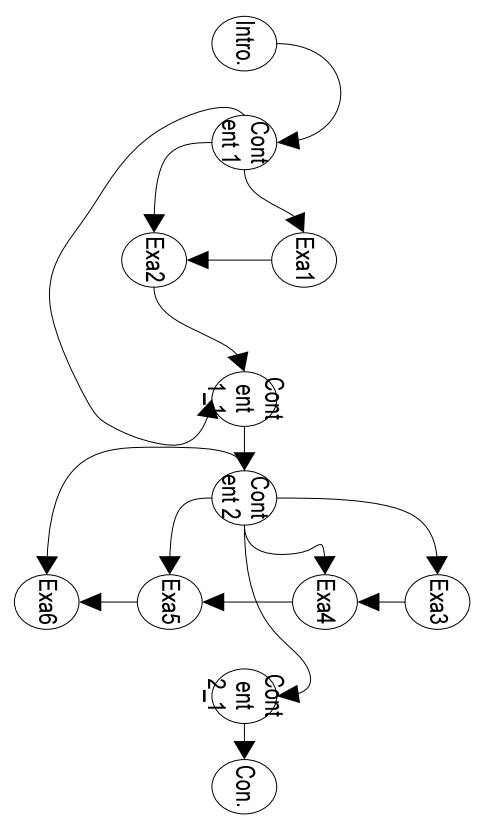

Figure 5. Learning objects graph.

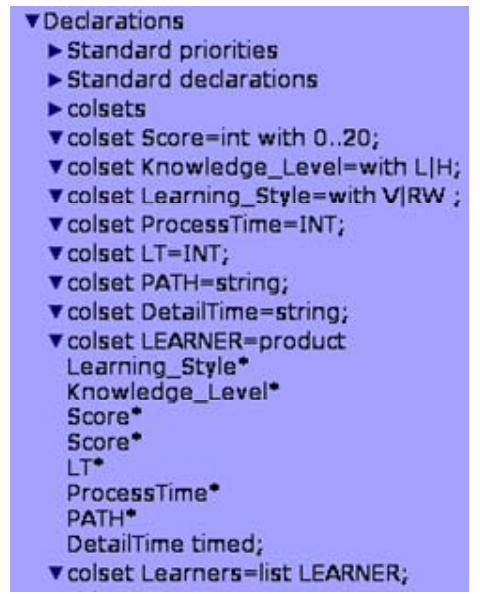

Figure 6. Declaration of color set in CPN tool.

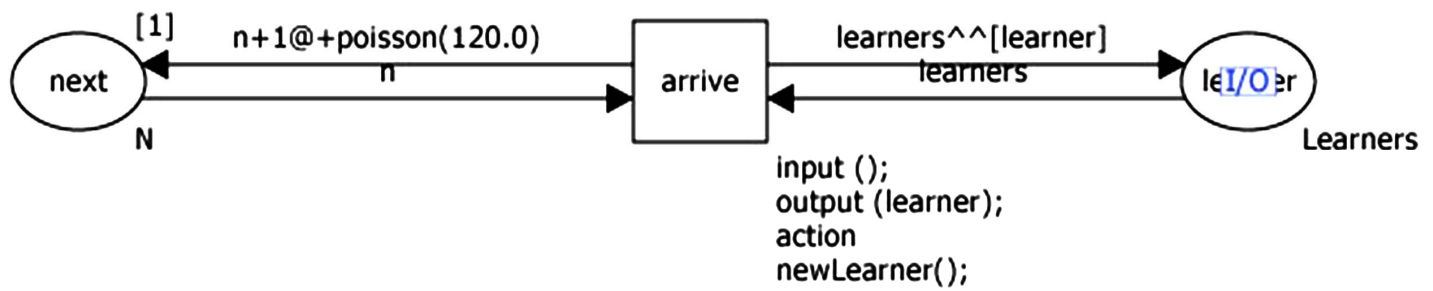

Figure 7. Arrival subpage. 
When a new learner arrives, the newLearner() function is used to create a token that represents a new learner. The declaration of the newLearner() function is depicted in Figure 8. When called, the newLearner() function returns a value from the LEARNER color set, i.e. it will return an 8-tuple as described above. The first component is the kind of the learning style, and using the Learning_Style.ran() function; it is chosen randomly. The second component is the kind of the knowledge level and using the Knowledge_Level.ran(); it is chosen randomly. The third and fourth components are scores in content 1 and 2 of book, respectively. The fifth component is arrive time and the sixth component is the amount of total time that learner remains in system. The seventh component is learning path and eighth component is detail time that learner remains in each learning unit. The type of queue is FIFO, so the new learners are added to end of queue by "learners ${ }^{\wedge}[$ learner]" arc inscription as depicts in Figure 7 and enter to system from first of queue by "learner:: learners" arc inscription in main page which is the toplevel page in the model as depicts in Figure 9.

The learning style of new learner is checked before entering to introduction node and based on its type; he/she is guided to 2 individual paths. These 2 paths are the same course, but their content, due to its style, are presented with different media. For example if learner is a Vstyle user, the media is flash files, audio and video files. So, the main page of the model as depicts in Figure 9 have 2 substitute transitions: "RWstyle" and "Vstyle" which are associated with 2 subpages with same name. The Vstyle transition guides learner to visual course; and RWstyle transition guides learners to text course based on their learning styles.

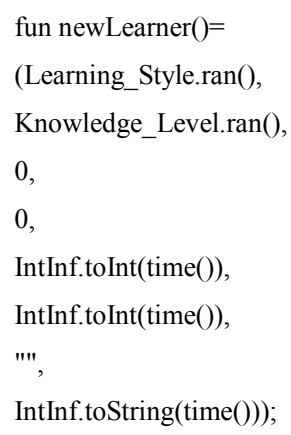

Figure 8. The declaration of the newlearner() function.

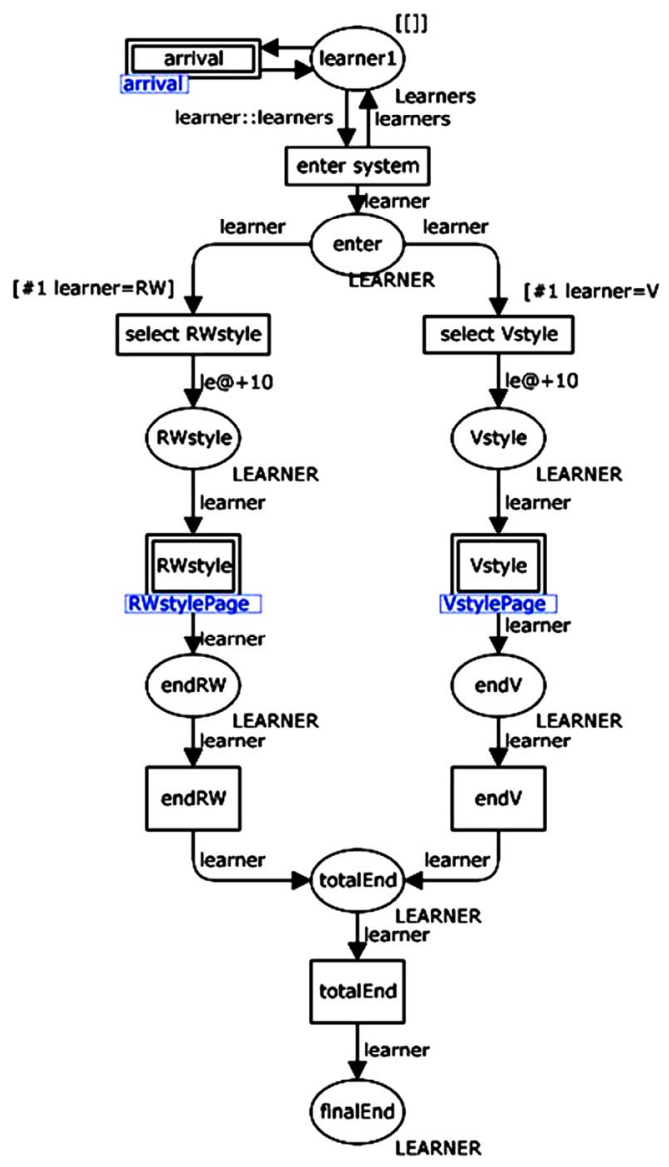

Figure 9. The main page. 
In both styles all learners (with any knowledge level) should pass introduction and content 1 ; based on course tutor's suggestion. Since the content 1 is the chapter which includes the test, the learners should test, after passing content1 (LO1). After that-based on his/her score and knowledge level-learner are guided to 4 different paths (because, in first decision making process based on learning style, after we determine the path, there are 2 factors which remain, so there are $2^{2}$ paths). These stages are illustrated in Figure $\mathbf{1 0}$ which is RWstyle subpage. For determining which paths (from 4 paths) is appropriate to learner; he/she enters to transition "decision making" and through this transition enters to the decision making subpage that is depicted in Figure 11.

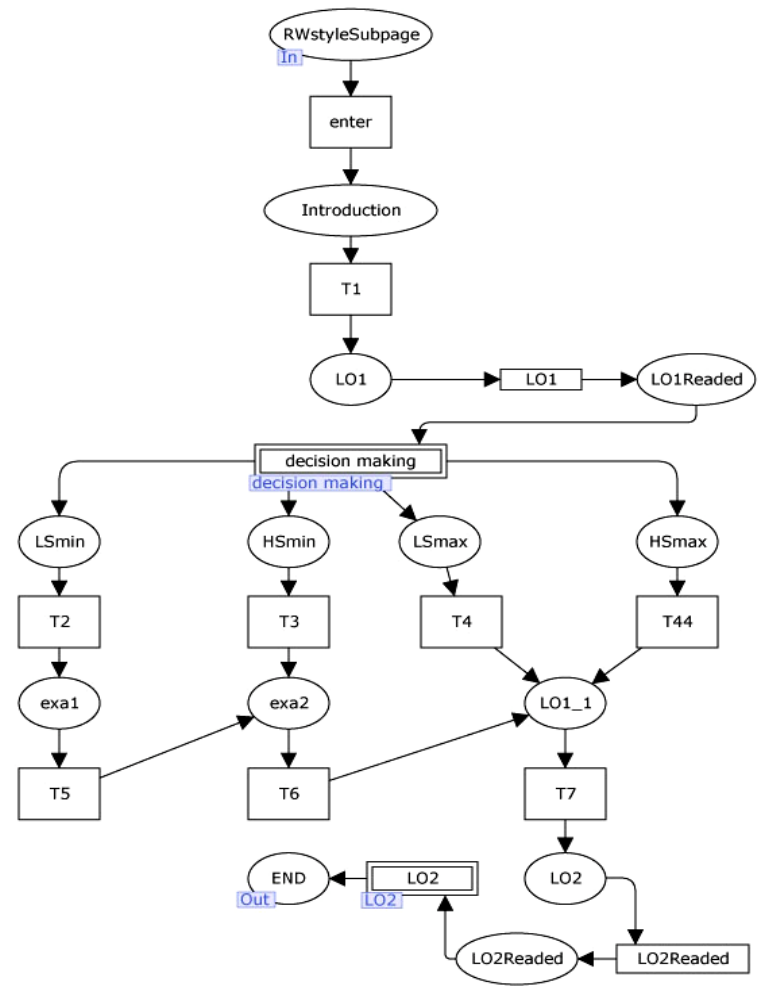

Figure 10. The RWstyle subpage.

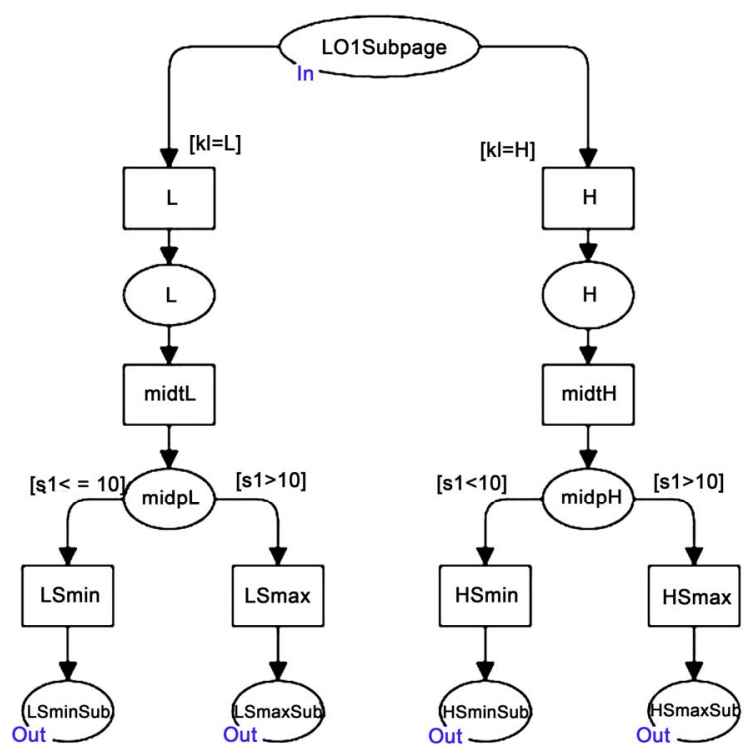

Figure 11. Decision making subpage. 
As you can see in the Figure 11, in subpage decision making, first system decides based on knowledge level and next decides based on score of content 1 . After that, the learner comes back to RWstyle page. In RWstyle page as depicted in Figure 10, for example if the learner's knowledge level is low and her/his score in content 1 is equal or less than 10 , learner should pass example1, example 2, content 1.1 (LO1_1), respectively. Whereas, if the learner's knowledge level is high and her/his score in content 1 is more than 10, learner doesn't need to pass the example 1 and example 2. After passing LO1-1, all learners (in 4 types) should pass content 2 . For this purpose, learners go to subpage LO2 by firing transition LO2. In subpage LO2, at first, based on knowledge level and score in LO1 by decision making11 subpage, which is similar to decision making subpage but does not call any function for assigning score, they divide. Next, learners go to decision making 2 subpage by firing transition with the same name as depicted in Figure 12. In subpage decision making 2-based on their score in LO2 as depicted in Figure 13-learners are guided to 8 individual paths. Next they come back to LO2 page and based on their situation (one of 8 types) pass different nodes to conclusion node.

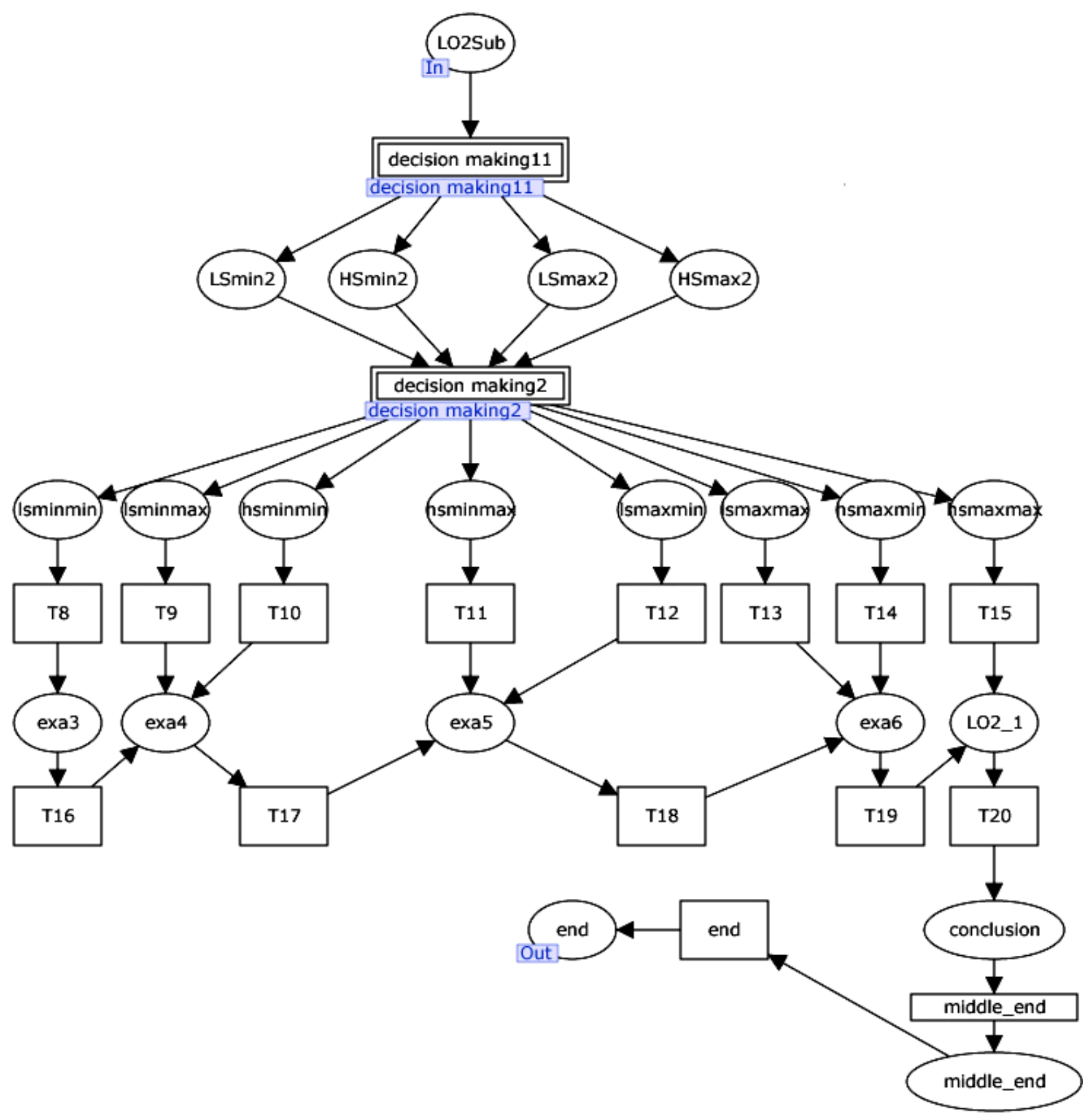

Figure 12. The LO2 subpage. 

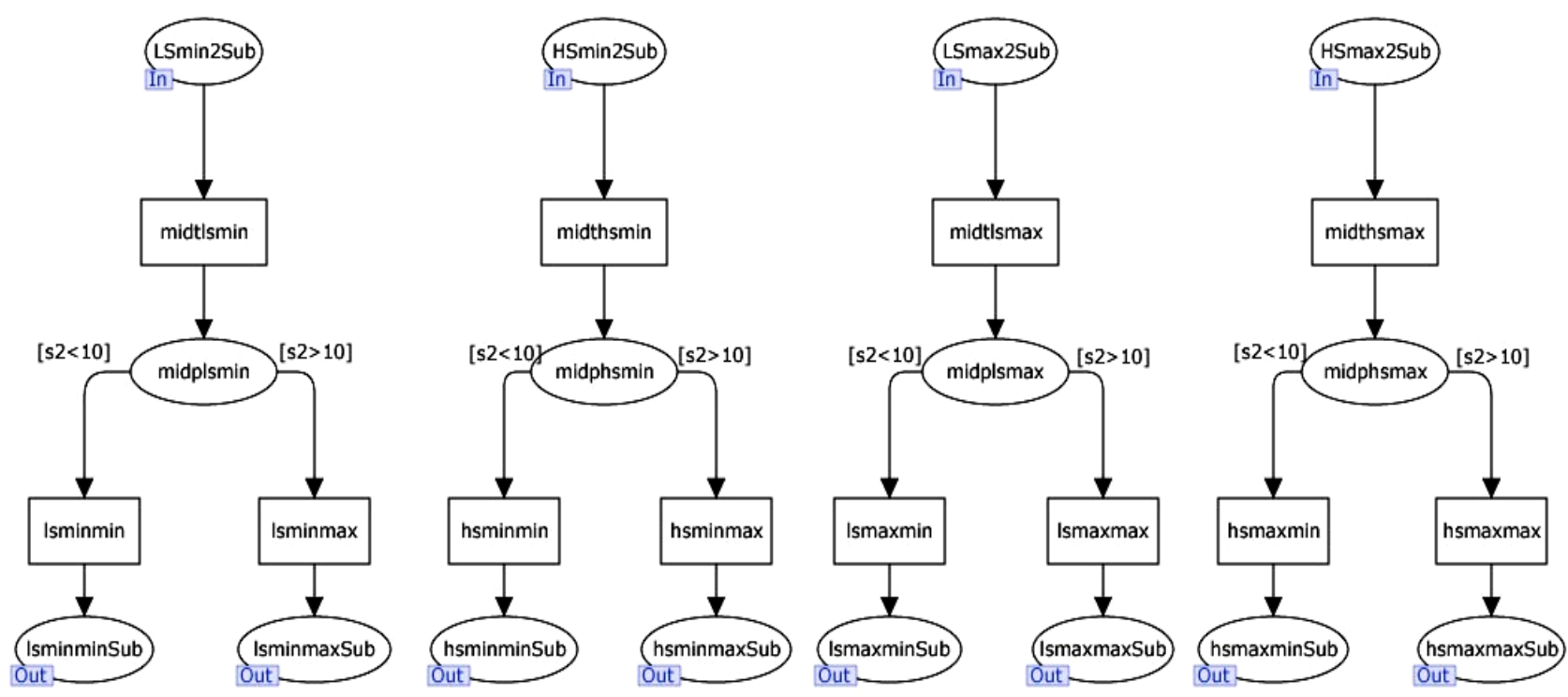

Figure 13. Decision making 2 subpage.

\section{Evaluation}

In the proposed system, to evaluate efficiency of the proposed method, as defined in Table 2, we compute response time for 3 types of users and next compare them with response time for learners in non adaptive system. In non adaptive system, users don't choose learning unit with awareness.

The purposes of considering these 3 types of users are: 1) we want to demonstrate if user has high knowledge level (type 1), does not need to pass some units of the course, and so she/he arrives to end section in shorter time, whereas user may pass unnecessary units in regular system; 2) We want to demonstrate, if user has low knowledge level but passes the course tests successfully (type 2), some learning units are ignored in his/her learning path and learner finishes course in shorter time whereas user may pass unnecessary units and does not pass necessary unit in regular system; 3) we want to demonstrate if learner has low knowledge level and does not pass current course learning tests with success, learner should pass all course units and can finish course in shorter time, whereas she/he may skip some units and spend more time in some units and this increases response time in non adaptive system.

For computing response time in non adaptive system, we delete guard condition of transitions in adaptive systems and allow learner choose arbitrary paths without constraints.

The simulation output in view of black box for adaptive system is depicted in Figure 14.

Each line of Figure 14 represented a learner. In each line, the first number is response time, the second number is learner number, the third number is step of simulation and fourth number is model time. For example in first line of Figure 14, first learner finished course in time 564 and it occurs in step 79 of simulation and model time has been 564. Since the Figure 14 is output in viewpoint of black box, type of learner, passed path and other details don't show.

For more detailed information, we use output in white box. The Figure 15 shows response time in viewpoint of white box. The information between each 2 lines is representing a learner.

Table 2. User types.

\begin{tabular}{|c|c|c|c|c|}
\hline $\begin{array}{l}\text { User } \\
\text { type }\end{array}$ & $\begin{array}{c}\text { Learning } \\
\text { style }\end{array}$ & \multicolumn{2}{|c|}{$\begin{array}{l}\text { Score in } \\
\text { content } 1\end{array}$} & $\begin{array}{c}\text { Score in } \\
\text { content } 2\end{array}$ \\
\hline Type 1 & $\mathrm{H}$ & \multicolumn{2}{|c|}{ more than 10} & more than 10 \\
\hline Type 2 & $\mathrm{~L}$ & \multicolumn{2}{|c|}{ more than 10} & more than 10 \\
\hline Type 3 & $\mathrm{~L}$ & \multicolumn{2}{|c|}{$\begin{array}{l}\text { equal or less } \\
\text { than } 10\end{array}$} & equal or less than 10 \\
\hline & ata & counter & step & time \\
\hline & & 1 & 79 & 564 \\
\hline & & 2 & 104 & 677 \\
\hline & & 3 & 120 & 767 \\
\hline & & 4 & 157 & 924 \\
\hline & & 5 & 189 & 1109 \\
\hline
\end{tabular}

Figure 14. Output in viewpoint of black box. 
The first parameter is learner's learning style, the second parameter is learner's knowledge level, the third parameter is learner's score in content 1 , the fourth parameter is learner score in content 2 , the fifth parameter is learner's arrive time, the sixth parameter is total time of waiting in queue and remaining in system, the seventh parameter is passed path by learner and eighth parameter is the time spent by learners in each unit. The number after parenthesis is response time. For example first line is representing a learner with RWstyle and high knowledge level and his/her score in content 1 is 15 and in content 2 is 14 . The arrive time for this user is 0 and total time is 564 . The passed path by him/her is :

“entersystem/selectRWstyle/Introduction/LO1/LO1

Testing/SelectHigh/selectLO1

Score $>10 / L O 1$ 1/LO2/LO2 Testing/SelectHigh/LO2 Score $>1 \overline{0} /$ Example6/LO2_1/conclusion"

And time spent by his/ her in each unit is:

$$
\text { "0/5/20/64/60/5/5/59/66/67/5/5/46/127/30" }
$$

In the time spent which is shown in above line, each number represents the time period of corresponding section in passed path. The number $5 \mathrm{~s}$ represent thinking times and other number represent time spent in learning units. For example the number 67 is representing the time of LO2 testing. Response time for learner which is subtraction of arrive time from total time (564-0) is 564. In addition, total time means 564, equal to summation of times that are shown in eighth parameter.

We summarize user types (is shown in Figure 14) and response time for adaptive and non adaptive system in Table 3.

Table 3. Response time for different users.

\begin{tabular}{ccc}
\hline User type & $\begin{array}{c}\text { Response time in } \\
\text { proposed system }\end{array}$ & $\begin{array}{c}\text { Response time in } \\
\text { non adaptive system }\end{array}$ \\
\hline Type 1 & 564 & 686 \\
Type 2 & 601 & 636 \\
Type 3 & 708 & 759 \\
\hline
\end{tabular}

Response Time in White Box

(RW, H, 15, 14, 0, 564, "enter system/select
RWstyle/Introduction/LO1/LO1 Testing/Select High/select LO1
Score>10/LO1_1/LO2/LO2 Testing/Select High/LO2 Scores
10/Example6/LO2
1/conclusion", " $0 / 5 / 20 / 64 / 60 / 5 / 5 / 59 / 66 / 67 / 5 / 5 / 46 / 127 / 30 ") 564$
"

(RW, H, 11, 14, 120, 677, "enter system/select

RWstyle/Introduction/LO1/LO1 Testing/Select High/select LO1

Score>10/LO1_1/LO2/LO2 Testing/Select High/LO2 Scores

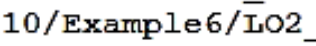

1/conclusion", " $\overline{1} 20 / 5 / 24 / 63 / 58 / 5 / 5 / 63 / 71 / 69 / 5 / 5 / 38 / 120 / 26 ") 557$
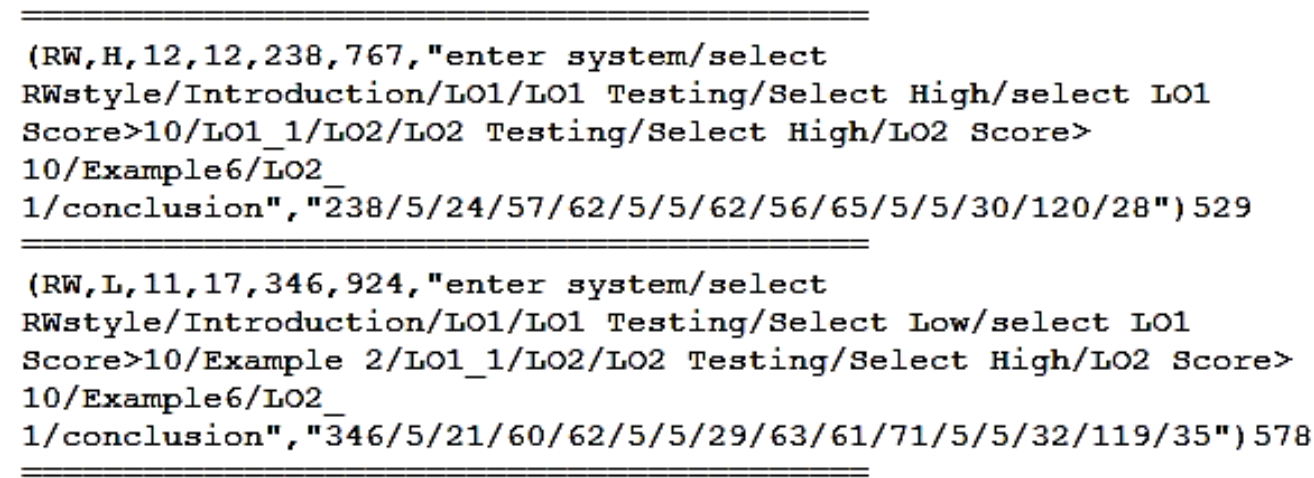

(V, H, 14, 13, 559, 1109, "enter system/select

Vstyle/Introduction/LO1/LO1 Testing/Select High/select LO1

Score>10/LO1_1/LO2/LO2 Testing/Select High/LO2 Score>

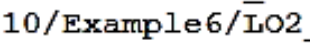

1/conclusion", "559/5/25/53/56/5/5/60/65/72/5/5/34/128/32") 550

Figure 15. Output in viewpoint of white box. 


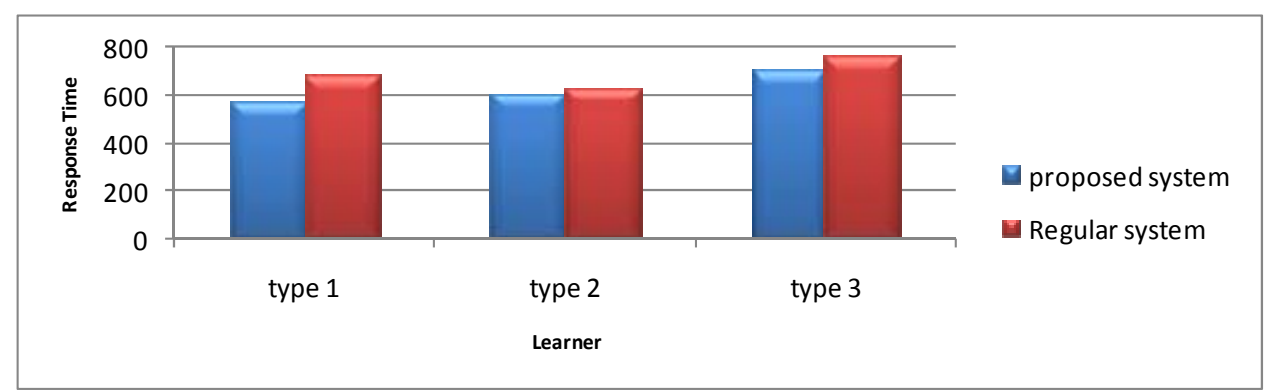

Figure 16. Time comparing for user types.

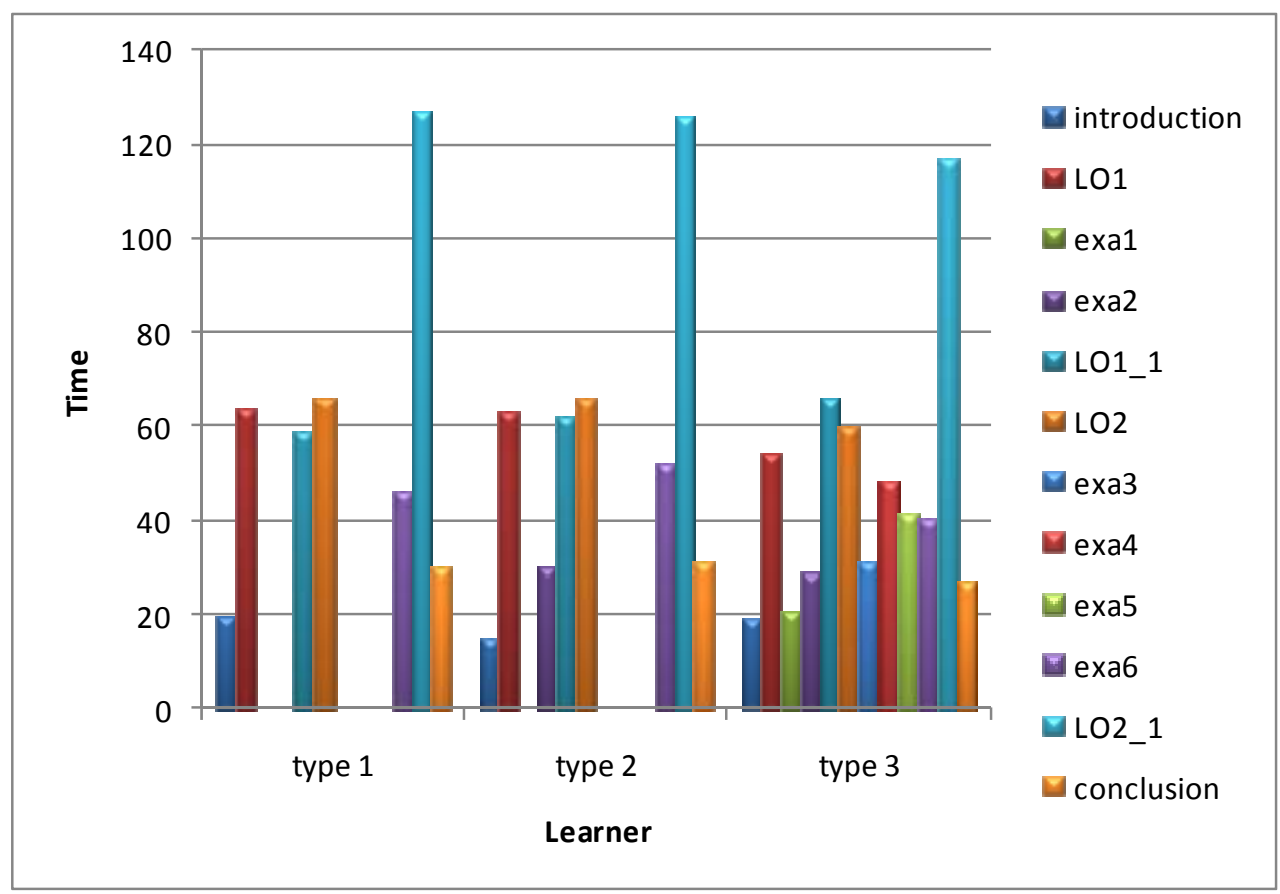

Figure 17. Detail time comparing for user types.

We compare response time for all learners in adaptive system with non adaptive system in Figure 16. As depicted in Figure 16 response time for all user type in adaptive system is shorter than users in non adaptive system.

The Figure 17 is representing spent time in different course units in adaptive system. Since the time of decision making in all cases is constant, we don't show them in Figure 17. Some of learners, because of their features, don't pass some units of course. But all learners pass main sections of course (Introduction, LO1, LO1_1, LO2, LO2 1, and conclusion). Some learners such as learner of type 3 is forced to pass all units of course as depicted in Figure 17.

\section{Conclusions and Future Work}

We proposed an adaptive method for e-learning systems by high level petri net. We use colored tokens for differ- entiating among learners with individual features. We use tokens with time stamp for computing time of tasks such as reading a learning unit or time of a test. We implement method for a sample course and analyze results. From 16 learner types in sample course, all of them have response time that was shorter than learner in non adaptive system. So, we can say this method increases performance.

This method has many advantages. For example formal semantic of petri net provides a formal method for adaptation. Also graphic presentation in petri net, facilitates understanding of method; even for unfamiliar people with petri net.

As future work, we want to examine proposed method in real course and analyze user's satisfaction and success. Also in many recommending systems such as search engine or electronic shop, in which user's individual features are important, this method can be used for adaptation. 


\section{REFERENCES}

[1] A. Dahbi, N. Elkamoun and A. Berraissoul, "Adaptation and Optimisation of Pedagogical Paths by Ants's Algorithm," ICTTA'06, 2nd Information and Communication Technologies, Damascus, October 2006, pp. 546-551.

[2] S. Gao and R. Dew, "Enhancing Web-Based Adaptive Learning with Colored Timed Petri Net," Lecture Notes in Computer Science, Vol. 4798, 2007, pp. 177-185. doi:10.1007/978-3-540-76719-0 20

[3] S. Gao, Z. Zhang and I. Hawryszkiewycz, "Supporting Adaptive Learning in Hypertext Environments: A High Level Timed Petri Net-Based Approach," International Journal of Intelligent Systems Technologies and Applications, Vol. 4, No. 3-4, 2008, pp. 341-354. doi:10.1504/IJISTA.2008.017277

[4] Y. Semet, E. Lutton and P. Collet, "Ant Colony Optimisation for E-Learning: Observing the Emergence of Pedagogic Suggestions," Proceedings of the 2003 IEEE Swarm Intelligence Symposium, Indianapolis, 24-26 April 2003, pp. 46-52.

[5] L. de Marcos, J. J. Martínez and J. A. Gutierrez, "Swarm Intelligence in E-Learning: A Learning Object Sequencing Agent Based on Competencies," Proceedings of the 10th Annual Conference on Genetic and Evolutionary Computation, Atlanta, 12-16 July 2008, pp. 17-24.

[6] F. Zhu and J. Cao, "Learning Activity Sequencing in Personalized Education System," Wuhan University Journal of Natural Sciences, Vol. 13, No. 4, 2008, pp. 461-465. doi:10.1007/s11859-008-0416-6

[7] C. M. Chen, H. M. Lee and Y. H. Chen, "Personalized E-Learning System Using Item Response Theory," Computers \& Education, Vol. 44, No. 3, 2005, pp. 237-255. doi:10.1016/j.compedu.2004.01.006

[8] N. Manouselis and D. Sampson, "Dynamic Knowledge Route Selection for Personalised Learning Environments Using Multiple Criteria," 20th IASTED International Conference in Applied Informatics, Innsbruck, 18-21 February 2002, pp. 448-453.

[9] J.-N. Chen, Y.-M. Huang and W. C.-C. Chu, "Applying Dynamic Fuzzy Petri Net to Web Learning System," Interactive Learning Environments, Vol. 13, No. 3, 2005, pp. 159-178. doi:10.1080/10494820500382810

[10] Y. C. Chang, Y. C. Huang and C. P. Chu, "B2 Model: A Browsing Behavior Model Based on High-Level Petri Nets to Generate Behavioral Patterns for E-Learning," Expert Systems with Applications, Vol. 36, No. 10, 2009, pp. 12423-12440. doi:10.1016/j.eswa.2009.04.044

[11] J. M. Su, S. S. Tseng, C. Y. Chen, J. F. Weng and W. N. Tsai, "Constructing SCORM Compliant Course Based on High-Level Petri Nets," Computer Standards \& Interfaces, Vol. 28, No. 3, 2006, pp. 336-355. doi:10.1016/j.csi.2005.04.001

[12] X. Q. Liu, M. Wu and J. X. Chen, "Knowledge Aggregation and Navigation High-Level Petri Nets-Based in ELearning," International Conference on Machine Learn- ing and Cybernetics, Vol. 1, 2002, pp. 420-425.

[13] R. M. Felder and L. K. Silverman, "Learning and Teaching Styles in Engineering Education," Engineering Education, Vol. 78, No. 7, 1988, pp. 674-681.

[14] P. García, A. Amandi, S. Schiaffino and M. Campo, "Evaluating Bayesian Networks' Precision for Detecting Students' Learning Styles," Computers \& Education, Vol. 49, No. 3, 2007, pp. 794-808. doi:10.1016/j.compedu.2005.11.017

[15] T. I. Wang, K. T. Wang and Y. M. Huang, "Using a StyleBased Ant Colony System for Adaptive Learning," Expert Systems with Applications, Vol. 34, No. 4, 2008, pp. 2449-2464. doi:10.1016/j.eswa.2007.04.014

[16] W. A. Drago and R. J. Wagner, "Vark Preferred Learning Styles and Online Education," Management Research News, Vol. 27, No. 7, 2004, pp. 1-13. doi:10.1108/01409170410784211

[17] T. Murata, "Petri Nets: Properties, Analysis and Applications," Proceedings of the IEEE, Vol. 77, No. 4, 1989, pp. 541-580.

[18] P. Abdulla and A. Nylén, "Timed Petri Nets and BQOs," Applications and Theory of Petri Nets, Vol. 2075, 2001, pp. 53-70. doi:10.1007/3-540-45740-2_5

[19] S. Christensen, L. Kristensen and T. Mailund, "Condensed State Spaces for Timed Petri Nets," Proceedings of the 22nd International Conference on Application and Theory of Petri Nets, Newcastle, 25-29 June 2001, pp. 101-120.

[20] R. S. Shaw, "A Study of Learning Performance of E-Learning Materials Design with Knowledge Maps," Computers \& Education, Vol. 54, No. 1, 2010, pp. 253-264. doi:10.1016/j.compedu.2009.08.007

[21] R. E. Walpole, R. H. Myers, S. L. Myers and K. Ye, "Probability and Statistics for Engineers and Scientists," 7th Edition, Prentice Hall, Upper Saddle River, 2002.

[22] L. Wells, "Performance Analysis Using Coloured Petri Nets," 10th IEEE International Symposium on Modeling, Analysis and Simulation of Computer and Telecommunications Systems, Fort Worth, 11-16 October 2002, pp. 217-221.

[23] K. Jensen, L. M. Kristensen and L. Wells, "Coloured Petri Nets and CPN Tools for Modelling and Validation of Concurrent Systems," International Journal on Software Tools for Technology Transfer, Vol. 9, No. 3-4, 2007, pp. 213-254. doi:10.1007/s10009-007-0038-x

[24] A. Ratzer, L. Wells, H. Lassen, M. Laursen, J. Qvortrup, M. Stissing, M. Westergaard, S. Christensen and K. Jensen, "CPN Tools for Editing, Simulating, and Analysing Coloured Petri Nets," Proceedings of the 24th International Conference on Applications and Theory of Petri Nets, Eindhoven, 23-27 June 2003, pp. 450-462.

[25] R. S. Shaw, "A Study of Learning Performance of ELearning Materials Design with Knowledge Maps," Computers \& Education, Vol. 54, No. 1, 2010, pp. 253-264. doi:10.1016/j.compedu.2009.08.007 